\title{
Analysis and Mitigation of Phase Noise in Centralized/De-centralized MIMO Systems*
}

\author{
Wei Zhang ${ }^{1}$, Xiujun Zhang ${ }^{2}$, Shidong Zhou ${ }^{3}$ and Jing Wang ${ }^{4}$ \\ ${ }^{1}$ Harbin Engineering University \\ 2,3,4 Tsinghua University \\ P.R.China
}

\section{Introduction}

The multiple-input multiple-output (MIMO) techniques are anticipated to be widely employed in future wireless networks to address the ever-increasing capacity demands. A MIMO channel, typically modeled as a matrix with independent and identically distributed (i.i.d.) complex Gaussian entries, provides multiple spatial dimensions for communications. At high signal-to-noise-ratio (SNR), Shannon capacity can increase linearly with the minimum number of transmit and receive antennas. However, achieving these dramatic capacity gains in practice, especially for outdoor deployment, could be problematic. One severe problem is the rank deficiency and ill-conditionness of the MIMO channel matrix $\mathrm{H}$. This is mainly caused by the spatial correlation due to the scattering environment and the antenna configurations, and sometimes by the "keyhole" effect even though the fading is essentially uncorrelated on each end of the channel. Therefore, the MIMO capacity may be greatly reduced and adding more (co-located) antennas only wastes resources.

The distributed antenna is an efficient way to solve this problem. In the downlink of a distributed antenna system, antennas allocated at several base stations (BS) constitute an antenna pool and can be used with a suitable MIMO transmission mode. Compared with a conventional centralized MIMO system, the distributed (de-centralized) MIMO system possesses many advantages, such as higher system capacity, better handoff performance, lower outage probability and higher data rates, especially at cell boundaries. However, it is also easier to be affected by phase noise (PN).

Oscillator noise stems from oscillator inaccuracies in both the transmitter and the receiver, and manifests itself in the baseband as additional phase and amplitude modulation of the received samples. The influence of oscillator noise on the signal depends on the noise characteristics of the oscillators in the system and on the signal bandwidth. It is generally split in amplitude noise and phase noise (PN). The influence of the amplitude noise on the data samples is often neglected (Robins, 1982). The phase noise contribution of both the transmitter and receiver can be viewed as an additional multiplicative effect of the radio channel, like fast and slow fading.

${ }^{*}$ The chapter is supported by the Fundamental Research Funds for the Central Universities in China and the China Postdoctoral Science Foundation funded project. 
In centralized MIMO systems, the presence of phase noise is an important limiting factor for a broadband system, i.e., a MIMO-OFDM system's performance, and depends on the quality and the operating conditions of the system's RF hardware. In conventional mobile radio systems around a carrier frequency of $2 \mathrm{GHz}$, the phase noise constitutes typically no severe limitation, however, when the carrier frequency goes up to a higher frequency, such as $15 \mathrm{GHz}$, its effects were less negligible and hence had to be investigated in more depth.

In distributed MIMO systems, system performance are limited by independent PN at local oscillators of $N_{t}$ transmit antennas and $N_{r}$ receive antennas. Some MIMO techniques, such as precoding, will suffer from independent $\mathrm{PN}$ as the orthogonality among sub-data-streams is destroyed. This involves with both narrowband and broadband MIMO systems.

This chapter will deal with the phase noise in centralized and decentralized (distributed) MIMO systems. The organization of this chapter is as follows.

Section 2 presents an overview of the phase noise effect and modeling of phase noise. PN can be modeled as a sampled Wiener Process for a free-running oscillator, and an Ornstein-Uhlenbeck Process (a Gaussian process with zero-mean and bounded variance) for a phase-locked loop (PLL) frequency synthesizer. In the following sections, we will consider effects of either/both of these two types of phase noise.

Section 3 addresses the influence of independent PN in centralized and distributed MIMO systems. We concentrate on narrowband systems applying linear precoding. In such a scenario, independent PN gives rise to SNR loss at the receiver and induces interference among sub-streams, and hence degrades the system performance. Then we discuss the feasible synchronization schemes for frequency and phase synchronization.

Simulation results are then given in Section 4 and finally Section 5 discusses the future research directions and concludes the chapter.

\section{Phase noise model}

In practice, a local oscillator (LO) can be realized with a free-running oscillator or with a PLL synthesizer. To characterize carrier imperfections, we use a random carrier time shift $\alpha(t)$ rather than the phase shift $\theta(t)$. A noisy LO output signal is then described as $x(t+\alpha(t))$ (Demir, 1998; Demir et al., 2000; Mehrotra, 1999; Petrovic et al., 2007). The phase and time shift at the carrier frequency $f_{c}$ are related by $\theta(t)=2 \pi f_{c} \alpha(t)$. To avoid confusion, in the following $\alpha(t)$ can also be called phase shift, phase perturbation, or phase noise.

\subsection{Free-running oscillators}

The main sources of oscillator noise are shot noise, thermal noise and flicker noise (also called $1 / f$ noise). The former two noise sources can be characterized as white Gaussian noise. The $1 / f$ noise is really a nonstationary process because it blows up at $f=0$. For analytical simplicity, a cut-off frequency is introduced, below which the spectrum deviates from $1 / f$ and attains a finite value at $f=0$, and thus the $1 / f$ noise can be modeled as a stationary and colored stochastic process (Demir, 1998). The power in such $1 / f$ noise source is concentrated at low frequencies, frequencies close to the carrier frequency.

Furthermore, the spectrum of the oscillator with colored noise sources has the shape of a Lorentzian around the carrier, and away from the carrier, the colored noise sources contribute terms that have a frequency dependence as $1 / f^{2}$ multiplied with the spectral density of the colored noise source, whereas the white noise sources contribute a term that has a $1 / f^{2}$ 
frequency dependence at remote frequencies. Unfortunately, up to now the $1 / f$ noise cannot be described in time domain with a mathematical form, for this reason in the following we only consider white Gaussian noise source.

If the noise source is white Gaussian, the time shift $\alpha(t)$ can be approximately described as a Wiener or Brownian motion process:

$$
\alpha(t)=\sqrt{c} B(t)
$$

where $c$ is a parameter describing the oscillator quality, and $B(t)$ stands for a standard Wiener process, i.e., an accumulated Gaussian random variable (r.v.) of unit variance. Therefore, $B(t)$ has the following properties:

1. $B(0) \sim \mathcal{N}(0,1)$;

2. $B\left(t_{1}\right)-B\left(t_{2}\right) \propto \sqrt{\left|t_{2}-t_{1}\right|} \times \mathcal{N}(0,1)$;

3. the variance of $B(t)$ increases linearly with time, i.e. $\sigma_{B}^{2}(t)=t$.

As such the variance of $\alpha(t)$ also increases linearly with time, i.e., $\sigma_{\alpha}^{2}(t)=c t$. In addition, the autocorrelation function of $\alpha(t)$ is given by

$$
E[\alpha(t) \alpha(t+\tau)]=c \min (t, t+\tau)
$$

Consider the relationship between Wiener process and normal distribution. We have

$$
\alpha(t)=\sqrt{c} \int_{0}^{t} \xi\left(t^{\prime}\right) d t^{\prime}
$$
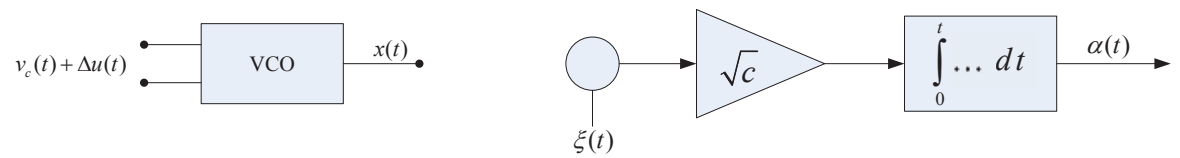

Fig. 1. Equivelent mathematical phase noise model of a free-running oscillator

Phase noise of both free-running oscillators and open-loop VCO can be described directly with (3), as shown in Fig. 1.

The constant $c$ cannot be directly obtained in practice. To evaluate the performance of an oscillator, we are usually interested in the power spectrum density (PSD) around the first harmonic, i.e. PSD for $f$ around $f_{c}$. Let $f_{m}$ denote the offset frequency related to carrier frequency $f_{c}$. For $0 \leq f_{m}<<f_{c}$, the single-sideband phase noise $\operatorname{PSD} \mathcal{L}\left(f_{m}\right)$ (in $\mathrm{dBc} / \mathrm{Hz}$ ) can be approximated as (Mehrotra, 1999)

$$
\mathcal{L}\left(f_{m}\right) \approx 10 \lg \left(\frac{f_{c}^{2} c}{\pi^{2} f_{c}^{4} c^{2}+f_{m}^{2}}\right)
$$

For $\pi f_{c}^{2} c<<f_{m}<<f_{c}, \mathcal{L}\left(f_{m}\right)$ can be approximated by

$$
\mathcal{L}\left(f_{m}\right) \approx 10 \lg \left(\left(\frac{f_{c}}{f_{m}}\right)^{2} c\right)
$$




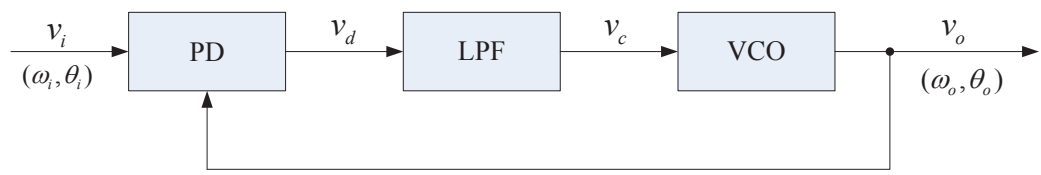

Fig. 2. PLL block diagram

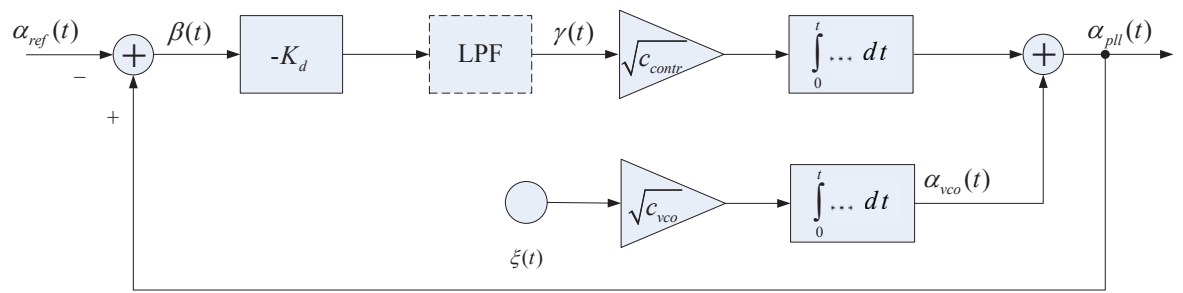

Fig. 3. Equivalent mathematical model of PLL phase noise

i.e., $\mathcal{L}\left(f_{m}\right)$ and $c$ are related by $\mathcal{L}\left(f_{m}\right) \approx 10 \log 10\left(c f_{c}^{2} / f_{m}^{2}\right)$. This approximation holds for $f_{m} \gg 3 \mathrm{~dB}$ bandwidth of phase noise (denoted by $\Delta f_{3 \mathrm{~dB}}$ ). As $\theta(t)=2 \pi f_{s} \alpha(t)$, Wiener process $\theta(t)$ can also be defined as $\sigma_{\theta}^{2}(t)=4 \pi \Delta f_{3 \mathrm{~dB}} t$, and

$$
\sigma_{\theta}^{2}(t)=4 \pi^{2} f_{s}^{2} \sigma_{\alpha}^{2}=4 \pi^{2} f_{s}^{2} c t=4 \pi \Delta f_{3 \mathrm{~dB}} t
$$

the constant $c$ is therefore related to $\Delta f_{3 \mathrm{~dB}}$ by $c=\Delta f_{3 \mathrm{~dB}} / \pi f_{c}^{2}$ (Petrovic et al., 2007). The time-sampling of the Wiener process $\alpha(t), \alpha[n]$, may ba represented in an integral-sum form

$$
\begin{aligned}
\alpha[n]=\alpha(n \cdot \Delta t) & =\sqrt{c} \sum_{i=0}^{n-1} \int_{0}^{\Delta t} \xi\left(t^{\prime}+i \cdot \Delta t\right) d t^{\prime} \\
& =\sqrt{c} \sum_{i=0}^{n-1} W_{i}(\Delta t) \\
& =\sqrt{c \cdot \Delta t} \sum_{i=0}^{n-1} w[i]
\end{aligned}
$$

where $W_{i}(\Delta t) \sim \mathcal{N}(0, \Delta t)$, and $w[i]$ is a Gaussian random variable, $w[i] \sim \mathcal{N}(0,1)$. The variance of $\alpha(n \Delta t)$ increases linearly with time, i.e., $\sigma_{\alpha}^{2}(n \Delta t)=c n \Delta t$.

\subsection{Phase-locked loop frequency synthesizer}

Consider the basic PLL circuit in Fig. 2. Let $\alpha_{r e f}(t), \alpha_{v c o}(t)$ and $\alpha_{p l l}(t)$ denote the PN of the reference signal, the PN of the open-loop VCO and the PN at the output of PLL circuit, respectively. $\alpha_{r e f}(t)$ and $\alpha_{v c o}(t)$ can also be modeled as Wiener process/one-dimensional Brown motion process. The equivalent phase noise model of a PLL is shown in Fig. 3. The input of the phase detector $(\mathrm{PD})$ is given by

$$
\beta(t)=\alpha_{p l l}(t)-\alpha_{r e f}(t)
$$


$\beta(t)$ is one component of a multidimensional Ornstein-Uhlenbeck process (Mehrotra, 2002). The correlation properties of $\beta(t)$ and $\alpha_{\text {ref }}(t)$ are

$$
\begin{aligned}
E\left[\beta\left(t_{1}\right) \alpha_{r e f}\left(t_{2}\right)\right] & =\sum_{i=1}^{n_{0}} \mu_{i} e^{\lambda_{i} \min \left\{0, t_{2}-t_{1}\right\}} \\
E\left[\beta\left(t_{1}\right) \beta\left(t_{2}\right)\right] & =\sum_{i=1}^{n_{0}} v_{i} e^{-\lambda_{i}\left|t_{2}-t_{1}\right|}
\end{aligned}
$$

where $n_{0}=1+o_{l p f}, o_{l p f}$ is the order of the low-pass filter. The calculation of parameters $\mu_{i}, v_{i}$ and $\lambda_{i}$ depends on the PLL circuit. The variance of the phase noise at the PLL output is then obtained by

$$
\begin{aligned}
\sigma_{\alpha_{p l l}}^{2}(t) & =E\left[\alpha_{p l l}^{2}(t)\right]-\left(E\left[\alpha_{p l l}(t)\right]\right)^{2} \\
& =E\left[\alpha_{p l l}^{2}(t)\right] \\
& =E\left[\left(\alpha_{r e f}(t)+\beta(t)\right)^{2}\right] \\
& =E\left[\alpha_{r e f}^{2}(t)+\beta^{2}(t)+2 \alpha_{r e f}(t) \beta(t)\right] \\
& =c_{r e f} t+\sum_{i=1}^{n_{0}}\left(2 \mu_{i}+v_{i}\right)
\end{aligned}
$$

The random differential equation of $\beta(t)$

$$
\begin{aligned}
\dot{\beta}(t) & =\dot{\alpha}_{p l l}(t)-\dot{\alpha}_{r e f}(t) \\
& =\sqrt{c_{\text {contr }}} \gamma(t)+\sqrt{c_{v c o}} \xi_{v c o}(t)-\sqrt{c_{r e f}} \xi_{r e f}(t)
\end{aligned}
$$

For the reference signal $\alpha_{r e f}(t)$ the PLL acts as a low-pass filter, whereas for the VCO signal the PLL acts like a high-pass filter. The $3 \mathrm{~dB}$ corner frequency for both filters is identical: $\omega_{G_{p} l}=$ $2 \pi f_{G_{p} l l}$. To minimize the phase noise at the PLL output, the corner frequency has to be chosen as high as possible. On the other hand, to keep the system stable, $\omega_{G_{p} l l}$ should not be higher than $1 / 10$ of the crystal frequency. The constants $c_{v c o}$ at the control node of the VCO can be calculated by $\sqrt{c_{v c o}}=\omega_{G_{p} l l} / K_{d}$, where $K_{d}$ is the given phase detector constant. Equation (12) is then rewritten as

$$
\beta(t)=\sqrt{c_{c o n t r}} \int_{0}^{t} \gamma\left(t^{\prime}\right) d t^{\prime}+\sqrt{c_{v c o}} \int_{0}^{t} \xi_{v c o}\left(t^{\prime}\right) d t^{\prime}-\sqrt{c_{r e f}} \int_{0}^{t} \xi_{r e f}\left(t^{\prime}\right) d t^{\prime}
$$

Sampling $\beta(t)$ with a frequency $1 / \Delta t$ and after some deviations, the discrete time model follows as

$$
\beta[n]=\beta[n-1]+\Delta t\left(\sqrt{c_{\text {contr }}} \gamma[n-1]+\sqrt{c_{v c o}} w_{v c o}-\sqrt{c_{r e f}} w_{r e f}\right)
$$

It can be shown that $\alpha_{p l l}(t)$ is asymptotically a zero-mean Gaussian process (Mehrotra, 2002), and the incremental random process $\alpha_{p l l}(t)-\alpha_{p l l}(t+\tau)$ has the mean

$$
\mu(t)=\lim _{t \rightarrow \infty}\left(\alpha_{p l l}(t)-\alpha_{p l l}(t+\tau)\right)=0
$$


and the variance

$$
\begin{aligned}
\sigma(t, \tau) & =\lim _{t \rightarrow \infty} E\left\{\left(\alpha_{p l l}(t)-\alpha_{p l l}(t+\tau)\right)^{2}\right\} \\
& =c_{r e f}|\tau|+2 \sum_{i=1}^{n_{0}}\left(\mu_{i}+v_{i}\right)\left(1-e^{-\lambda_{i}|\tau|}\right)
\end{aligned}
$$

which is independent of $t$. Therefore, the cumulative phase-noise increment between samples $l$ and $k$ of the received signal, $\Delta \theta_{k l}=2 \pi f_{c}\left(\alpha_{p l l}(k \Delta t)-\alpha_{p l l}(l \Delta t)\right)$, has the characteristic function (Petrovic et al., 2007)

$$
E\left\{e^{j \Delta \theta_{k l}}\right\}=e^{-\frac{\omega_{0}^{2}}{2}\left(c_{r e f}|k-l| \Delta t+2 \sum_{i=1}^{n_{0}}\left(\mu_{i}+v_{i}\right)\left(1-e^{-\lambda_{i}|k-l| \Delta t}\right)\right)}
$$

Algorithms for the calculation of PLL phase noise PSD are also given in literature (Mehrotra, 2002).

\section{Phase noise in MIMO systems}

\subsection{MIMO system model}

Consider a narrowband MIMO system with $N_{T}$ transmit antennas and $N_{R}$ receive antennas, as shown in Fig. 4. The system transmission model is expressed as (Tse \& Viswanath, 2004)

$$
\mathrm{y}=\mathrm{Hx}+\mathrm{w}
$$

where $\mathrm{x}=\left[x_{0}, x_{1}, \ldots, x_{N_{T}-1}\right]^{T}, \mathrm{x} \in \mathcal{C}^{N_{T}}$ and $\mathrm{y}=\left[y_{0}, y_{1}, \ldots, y_{N_{R}-1}\right]^{T}, \mathrm{y} \in \mathcal{C}^{N_{R}}$ represent transmit signal vector and receive signal vector at a symbol time, respectively (the time index is dropped for simplicity). $\mathrm{H}=\left[h_{i j}\right]_{N_{R} \times N_{T}}, \mathrm{H} \in \mathcal{C}^{N_{R} \times N_{T}}$ denotes channel matrix, where $h_{i j}$ represents the channel gain from transmitted antenna $j$ to received antenna $i$, and is an independent and identically distributed complex Gaussian random variable of zaro mean and unity variance, i.e., $h_{i j} \sim \mathcal{C N}(0,1)$. $\left|h_{i j}\right|$ is Rayleigh distributed and $\left|h_{i j}\right|^{2}$ is exponentially distributed with a unity mean. $\mathrm{w}=\left[w_{0}, w_{1}, \ldots, w_{N_{R}-1}\right]^{T}$ denotes an $N_{r}$-dimensional complex Gaussian random vector with zero mean and variance $N_{0}$, i.e. $w \sim \mathcal{C} \mathcal{N}\left(0, N_{0} I_{N_{R}}\right)$, where I is identity matrix.

\subsubsection{Precoding based on singular value decomposition}

Assuming the channel is known at the transmitter, the singular value decomposition (SVD) of the channel matrix $\mathrm{H}$

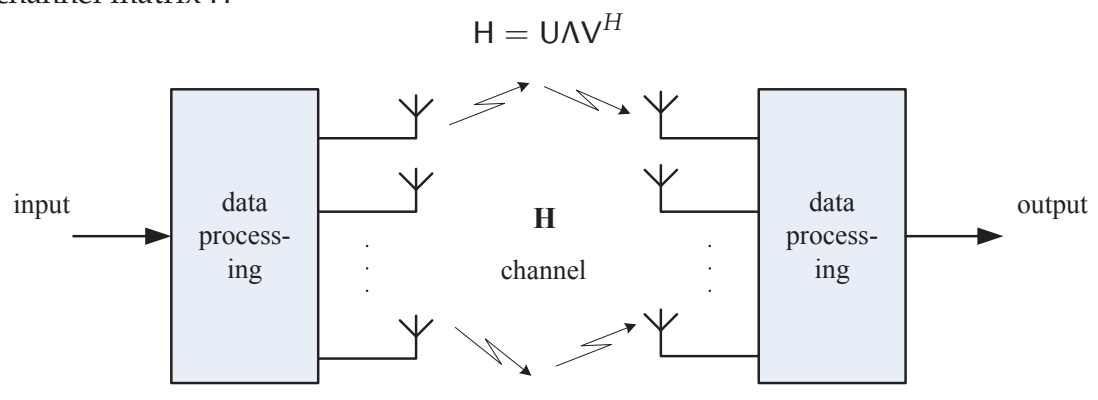

Fig. 4. MIMO system 
where

$$
\Lambda=\left(\begin{array}{cc}
\Lambda_{1} & 0 \\
0 & 0
\end{array}\right)
$$

and the diagonal elements $\Lambda_{1}=\operatorname{diag}\left\{\left[\lambda_{1}, \lambda_{2}, \ldots, \lambda_{r}\right]\right\}$ are the ordered singular values of the matrix $\mathrm{H}$, where

$$
\lambda_{1} \geq \lambda_{2} \geq \ldots \geq \lambda_{r}>0, \quad r=\operatorname{rank}(\mathrm{H})
$$

and the rank of $\mathrm{H}, r \leq \min \left(N_{T}, N_{R}\right)$. In (19), $\mathrm{U}$ and $\mathrm{V}$ are $N_{R} \times N_{R}$ and $N_{T} \times N_{T}$ unitary matrices, respectively, and satisfy $\mathrm{UU}^{H}=\mathrm{I}_{N_{R}}$ and $\mathrm{VV}^{H}=\mathrm{I}_{N_{T}}, \mathrm{U} \in \mathcal{C}^{N_{R} \times N_{R}}, \mathrm{~V} \in \mathcal{C}^{N_{T} \times N_{T}}$. Since

$$
\mathrm{HH}^{H}=\mathrm{U} \wedge \wedge^{T} \mathrm{U}^{H}
$$

The squared singular values $\lambda_{i}^{2}$ are the eigenvalues of the matrix $\mathrm{HH}^{H}$ and also of $\mathbf{H}^{H} \mathrm{H}$. The SVD can be rewritten as

$$
\mathbf{H}=\sum_{i=1}^{r} \lambda_{i} \mathbf{u}_{i} \mathbf{v}_{i}^{H}
$$

i.e., the sum of rank-one matrices $\lambda_{i} u_{i} v_{i}^{H}$ 's. It can be seen that the rank of $H$ is precisely the number of non-zero singular values.

If we define

$$
\begin{aligned}
\tilde{y} & :=U^{H} \mathrm{y} \\
\tilde{x} & :=\mathrm{V}^{H} \mathrm{x} \\
\tilde{\mathrm{w}} & :=\mathrm{U}^{H} \mathrm{w}
\end{aligned}
$$

then we can rewrite the channel (18) as

$$
\tilde{y}=U^{H} H V \tilde{x}+\tilde{w}=\Lambda \tilde{x}+\tilde{w}
$$

where $\tilde{w} \sim \mathcal{C N}\left(0, N_{0} I_{N_{R}}\right)$ has the same distribution as $w$ and $\|\tilde{x}\|^{2}=\|x\|^{2}$. Thus, the energy is preserved and we have an equivalent representation as a parallel of Gaussian channels (as shown in Fig. 5)

$$
\tilde{y}_{i}=\lambda_{i} \tilde{x}_{i}+\tilde{w}_{i}, \quad i=1,2, \ldots, r .
$$

where $x_{i} \sim \mathcal{C N}\left(0, \sigma_{x}^{2}\right)$. The data transmitted on such a Gaussian channel construct a data sub-stream. If the power is equally assigned to all transmit antennas and $\mathrm{H}$ is the

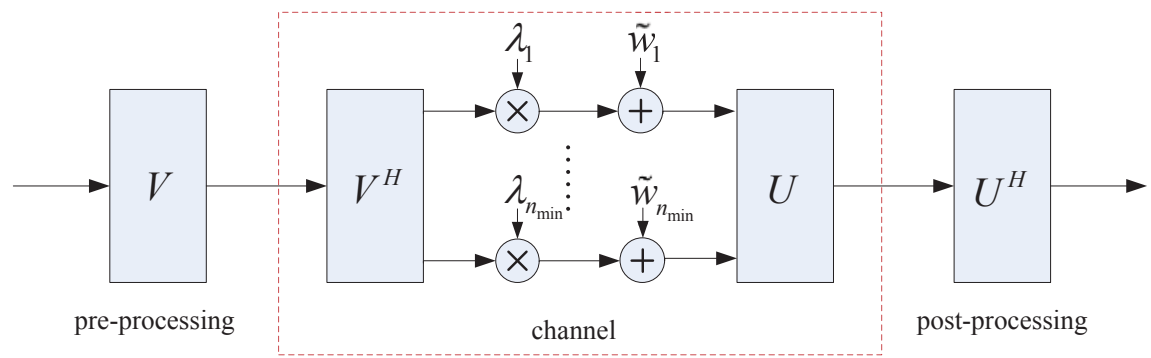

Fig. 5. Singular value decomposition of a MIMO channel 
time-invariant deterministic channel, then

$$
\sum_{i=1}^{r} \lambda_{i}^{2}=\operatorname{Tr}\left[\mathrm{HH}^{H}\right]=\sum_{i, j}\left|h_{i j}\right|^{2} .
$$

If $\mathrm{H}$ is Rayleigh fading channel, and $h_{i j} \sim \mathcal{C N}(0,1)$, then

$$
E\left[\sum_{i=1}^{r} \lambda_{i}^{2}\right]=E\left[\operatorname{Tr}\left[\mathrm{HH}^{H}\right]\right]=\sum_{i, j} E\left[\left|h_{i j}\right|^{2}\right]=N_{R} \times N_{T}
$$

It follows that

$$
E\left[\lambda_{i}^{2}\right]=N_{R} N_{T} / r
$$

\subsubsection{MIMO system with phase noise}

In decentralized (distributed) MIMO systems, antennas are placed in different positions in a cell, and can be seen as being mutually independent. Signals received by different antennas are transmitted to a central processing unit via optical fiber. To minimize the cost, the RF unit and the antenna(s) should be placed together. When multiple remote antennas form a virtual cell, RF signals of different antennas have independent clocks. Given a MIMO system using precoding based on SVD, in the following we will investigate the impacts of the dependent and independent clocks on system performance.

Consider the narrowband MIMO system in Fig. 4. When there exists phase noise, we rewrite (25) as

$$
\tilde{y}=U^{H} \Theta H \Phi V \tilde{x}+\tilde{w}
$$

where $\Phi=\operatorname{diag}\left\{\left[e^{j \phi_{1}}, e^{j \phi_{2}}, \ldots, e^{j \phi_{N_{T}}}\right]\right\}$ and $\Theta=\operatorname{diag}\left\{\left[e^{j \theta_{1}}, e^{j \theta_{2}}, \ldots, e^{j \theta_{N_{R}}}\right]\right\} .\left[\phi_{1}, \phi_{2}, \ldots, \phi_{N_{T}}\right]$ denote the phase noise related to different transmit antennas, whereas $\left[\theta_{1}, \theta_{2}, \ldots, \theta_{N_{R}}\right]$ denote the phase noise related to different receive antennas.

\subsection{Phase noise in centralized MIMO systems}

In a centralized MIMO system, multiple transmit/receive antennas are placed together with the same transmitter/receiver and so we have $\phi_{1}=\phi_{2}=\ldots=\phi_{N_{T}}=\phi$ and $\theta_{1}=\theta_{2}=$ $\ldots=\theta_{N_{R}}=\theta$. That is to say, at any instant phase noises at transmitter(s)/receiver(s) are fully correlated, but $\phi$ and $\theta$ are statistically independent. Therefore, (30) can be simplified to be

$$
\tilde{y}=e^{j(\phi+\theta)} \Lambda \tilde{x}+\tilde{w}
$$

This is equivalent to the case of point-to-point communications: in transmitters, phase noise can result in adjacent-channel interference and modulation errors; in receivers, phase noise can result in demodulation errors and degraded sensitivity and dynamic range, such as the rotation of signal constellation, and further degrade the system bit error rate (BER) performance. The specification, calculation, and reduction of oscillator noise are therefore of great importance in wireless system design (Rohde \& Netwkirk, 2000). 


\subsection{Phase noise in decentralized MIMO systems}

\subsubsection{Identical transmitter clock, independent receiver clocks}

Consider an uplink transmission in a distributed MIMO system, where all transmit antennas share the clock and distributed receive antennas have independent clocks. We have then $\phi_{1}=$ $\phi_{2}=\ldots=\phi_{N_{T}}=\phi$ and $\theta_{1} \neq \theta_{2} \neq \ldots \neq \theta_{N_{R}}$, i.e., phase noises of different RF units are mutually independent. Equation (30) can be simplified to be

$$
\begin{aligned}
\tilde{y} & =e^{j \phi} U^{H} \Theta H \vee \tilde{x}+\tilde{w} \\
& =e^{j \phi} U^{H} \Theta U \wedge \tilde{x}+\tilde{w}
\end{aligned}
$$

Precoding can be regarded as a generalization of beamforming to support multi-layer transmission in multi-antenna wireless communications. Independent receiver phase noises not only reduce the signal power, but also result in interference among sub-streams. Assuming $r=N_{R} \leq N_{T}$, the received signals of the $i$ th data sub-stream are given by

$$
\tilde{y}_{i}=\lambda_{i} \tilde{x}_{i} \sum_{l=1}^{N_{R}}\left|u_{l i}\right|^{2} e^{j\left(\theta_{l}+\phi\right)}+\sum_{k=1, k \neq i}^{r} \lambda_{k} \tilde{x}_{k} \sum_{l=1}^{N_{R}} u_{l i}^{*} u_{l k} e^{j\left(\theta_{l}+\phi\right)}+\tilde{w}_{i},
$$

where the first term on the right hand side (RHS) of (33) is the desired signal, the second term is the interference introduced by phase noises. Since matrix $U$ is unitary, $h_{i j} \sim \mathcal{C N}(0,1)$ and $h_{i j}$ is independent of phase noise, it follows that the element $\left|u_{l i}\right|^{2}$ is independent and identically distributed random variable, and satisfies

$$
\begin{aligned}
& \sum_{l=1}^{N_{R}}\left|u_{l i}\right|^{2}=1 \\
& \sum_{l=1}^{N_{R}} u_{l k}^{*} u_{l j}=0, \text { for } j \neq k
\end{aligned}
$$

According to the law of large numbers,

$$
N_{R} \times \frac{1}{N_{R}} \sum_{l=1}^{N_{R}}\left|u_{l i}\right|^{2} e^{j\left(\theta_{l}+\phi\right)} \longrightarrow N_{R} \times E\left[\left|u_{l i}\right|^{2}\left(\cos \left(\theta_{l}+\phi\right)+j \sin \left(\theta_{l}+\phi\right)\right)\right]
$$

Assume $\theta_{l}$ to be symmetrically distributed about 0 , then $E\left[\sin \theta_{l}\right]=0$. Further we have

$$
\begin{aligned}
E\left[\cos \left(\theta_{l}+\phi\right)\right] & =E\left[\cos \theta_{l} \cos \phi+\sin \theta_{l} \sin \phi\right]=E\left[\cos \theta_{l}\right] \cos \phi \\
E\left[\sin \left(\theta_{l}+\phi\right)\right] & =E\left[\sin \theta_{l} \cos \phi+\cos \theta_{l} \sin \phi\right]=E\left[\cos \theta_{l}\right] \sin \phi
\end{aligned}
$$

and from (34) we get

$$
\sum_{l=1}^{N_{R}}\left|u_{l i}\right|^{2}=1 \quad \Longrightarrow \quad \frac{1}{N_{R}} \sum_{l=1}^{N_{R}}\left|u_{l i}\right|^{2}=\frac{1}{N_{R}}
$$

when $N_{R} \longrightarrow \infty$,

$$
\frac{1}{N_{R}} \sum_{l=1}^{N_{R}}\left|u_{l i}\right|^{2}=\frac{1}{N_{R}} \quad \Longrightarrow \quad E\left[\left|u_{l i}\right|^{2}\right]=\frac{1}{N_{R}}
$$


The RHS of (35) is then simplified to be

$$
\begin{aligned}
& N_{R} \times E\left[\left|u_{l i}\right|^{2}\left(\cos \left(\theta_{l}+\phi\right)+j \sin \left(\theta_{l}+\phi\right)\right)\right] \\
= & N_{R} \times E\left[\left|u_{l i}\right|^{2}\right] E\left[\cos \left(\theta_{l}+\phi\right)+j \sin \left(\theta_{l}+\phi\right)\right] \\
= & E\left[\cos \theta_{l}\right] e^{j \phi}
\end{aligned}
$$

Therefore,

$$
\left\|\sum_{l=1}^{N_{R}}\left|u_{l i}\right|^{2} e^{j\left(\theta_{l}+\phi\right)}\right\|^{2}=\left(E\left[\cos \theta_{l}\right]\right)^{2}
$$

The power of the desired signal

$$
P_{R, i}=\left\|\lambda_{i} \tilde{x}_{i} \sum_{l=1}^{N_{R}}\left|u_{l i}\right|^{2} e^{j\left(\theta_{l}+\phi\right)}\right\|^{2}=\lambda_{i}^{2}|\tilde{x}|^{2}\left(E\left[\cos \theta_{l}\right]\right)^{2}
$$

Consider only the part of $\mathrm{U}^{H} \Theta U$ in (32). Denote $\mathrm{C}=\mathrm{U}^{H} \Theta U$. Since $\mathrm{U}^{H} \mathrm{U}=\mathrm{UU}^{H}=\mathrm{I}$ and $\left|e^{\theta_{i}}\right|=$ 1 , the diagonal entries of $\Theta, e^{j \theta_{1}}, e^{j \theta_{2}}, \ldots, e^{j \theta_{N_{R}}}$, are eigenvalues of $C$, and the eigenvalue of $C^{H}$ is 1 . That is to say, on either of parallel Gaussian channels the sum of the interference power to other subchannels and desired signals equals to 1 (the square of $\lambda$ is not counted).Then the average power of the desired signals in (33) is given by

$$
\begin{aligned}
E\left[P_{R, i}\right] & =E\left[\lambda_{i}^{2} \tilde{x}_{i} \tilde{x}_{i}^{*} \sum_{l=1}^{N_{R}}\left|u_{l i}\right|^{2} e^{j\left(\theta_{l}+\phi\right)} \sum_{m=1}^{N_{R}}\left|u_{m i}\right|^{2} e^{-j\left(\theta_{m}+\phi\right)}\right] \\
& =E\left[\lambda_{i}^{2}\right] E\left[\left|x_{i}\right|^{2}\right]\left(\frac{1}{N_{R}}+\frac{N_{R}\left(N_{R}-1\right)}{2} E\left[\left|u_{l i}\right|^{2}\left|u_{m i}\right|^{2} \cdot 2 \Re\left(e^{j\left(\theta_{l}-\theta_{m}\right)}\right)\right]\right) \\
& =\frac{N_{T} N_{R} P}{r^{2}}\left(\frac{1}{N_{R}}+\frac{N_{R}-1}{N_{R}} E\left[\cos \left(\theta_{l}-\theta_{m}\right)\right]\right) \\
& =\frac{N_{T} N_{R} P}{r^{2}}\left(\frac{1}{N_{R}}+\frac{N_{R}-1}{N_{R}} E\left[\cos \theta_{l} \cos \theta_{m}-\sin \theta_{l} \sin \theta_{m}\right]\right) \\
& =\frac{N_{T} N_{R} P}{r^{2}}\left(\frac{1}{N_{R}}+\frac{N_{R}-1}{N_{R}} E\left[\cos \theta_{l}\right] E\left[\cos \theta_{m}\right]\right) \\
& =\frac{N_{T} P}{r^{2}}\left(1+\left(N_{R}-1\right)\left(E\left[\cos \theta_{l}\right]\right)^{2}\right)
\end{aligned}
$$

where $E\left[|x|^{2}\right]=P / r$ is assumed, the average channel gain $E\left[\lambda_{k}^{2}\right]=E\left[\lambda_{i}^{2}\right]=N_{R} N_{T} / r$, and $\theta_{l}$ is symmetrically distributed about 0 . The average interference power from a parallel channel to other channels is given by

$$
E\left[P_{I, i}\right]=E\left[\lambda_{i}^{2}\right] E\left[|x|^{2}\right]-E\left[P_{R}\right]=\frac{N_{T} P}{r^{2}}\left(N_{R}-1\right)\left(1-\left(E\left[\cos \theta_{l}\right]\right)^{2}\right)
$$

Define $\gamma_{0}=P / N_{0}$. The signal-to-interference-and-noise-ratio (SINR) is then calculated by

$$
\operatorname{SINR}_{i}=\frac{E\left[P_{R, i}\right]}{E\left[P_{I, i}\right]+N_{0}}=\frac{1+\left(N_{R}-1\right)\left(E\left[\cos \theta_{l}\right]\right)^{2}}{\left(N_{R}-1\right)\left(1-\left(E\left[\cos \theta_{l}\right]\right)^{2}\right)+r^{2} /\left(N_{T} \gamma_{0}\right)}
$$

It is shown that the independent phase noises worsen the received SNR and degraded the overall performance of the communication systems. 


\subsubsection{Identical receiver clock, independent transmitter clocks}

Consider a downlink scenario where one mobile terminal receive signals from multiple cell antennas. Now $\theta_{1}=\theta_{2}=\ldots=\theta_{N_{R}}=\theta$, and without the loss of generality, assuming $\phi_{1} \neq \phi_{2} \neq \ldots \neq \phi_{N_{T}}$ (30) is simplified to be

$$
\begin{aligned}
\tilde{y} & =e^{j \theta} U^{H} \mathrm{H} \Phi \mathrm{V} \tilde{x}+\tilde{w} \\
& =e^{j \theta} \Lambda \mathrm{V}^{H} \Phi \mathrm{V} \tilde{x}+\tilde{\mathbf{w}}
\end{aligned}
$$

Similarly, the signal received on $i$ th parallel channel is expressed as

$$
\tilde{y}_{i}=\lambda_{i} \tilde{x}_{i} \sum_{l=1}^{N_{T}}\left|v_{l i}\right|^{2} e^{j\left(\phi_{l}+\theta\right)}+\lambda_{i} \sum_{k=1, k \neq i}^{r} \tilde{x}_{k} \sum_{l=1}^{N_{T}} v_{l i}^{*} v_{l k} e^{j\left(\phi_{l}+\theta\right)}+\tilde{w}_{i},
$$

Also we get

$$
\begin{aligned}
E\left[P_{R, i}\right] & =\frac{E\left[\lambda_{i}^{2}\right] E\left[|x|^{2}\right]}{N_{T}}\left(1+\left(N_{T}-1\right)\left(E\left[\cos \phi_{l}\right]\right)^{2}\right) \\
& =\frac{N_{R} P}{r^{2}}\left(1+\left(N_{T}-1\right)\left(E\left[\cos \phi_{l}\right]\right)^{2}\right)
\end{aligned}
$$

and

$$
\operatorname{SINR}=\frac{E\left[P_{R, i}\right]}{E\left[P_{I, i}\right]+N_{0}}=\frac{1+\left(N_{T}-1\right)\left(E\left[\cos \phi_{l}\right]\right)^{2}}{\left(N_{T}-1\right)\left(1-\left(E\left[\cos \phi_{l}\right]\right)^{2}\right)+r^{2} /\left(N_{R} \gamma_{0}\right)}
$$

\subsubsection{Independent transmitter/receiver clocks}

From the analysis above, we can find out that with the increase of phase noise, the SINR degrades rapidly. Therefore, for a more generalized scenario where RF units of all transmit/receive antennas have independent clocks, only the research on the condition that $\theta<<1$ and $\phi<<1$ is meaningful. Under these assumptions, we have $e^{j \theta_{l}} \approx 1+j \theta_{l}$ and $e^{j \phi_{m}} \approx 1+j \phi_{m}$, and (30) can be simplified to be

$$
\tilde{y} \approx \Lambda \tilde{x}+j U^{H} \bar{\Theta} H \bigvee \tilde{x}+j U^{H} H \bar{\Phi} \vee \tilde{x}-U^{H} \bar{\Theta} H \bar{\phi} \vee \tilde{x}+\tilde{w}
$$

where $\bar{\Phi}=\operatorname{diag}\left\{\left[\phi_{1}, \phi_{2}, \ldots, \phi_{N_{T}}\right]\right\}, \bar{\Theta}=\operatorname{diag}\left\{\left[\theta_{1}, \theta_{2}, \ldots, \theta_{N_{R}}\right]\right\}$. When $\theta_{l} \longrightarrow 0$ and $\phi_{l} \longrightarrow 0$, the fourth term on the RHS of (49) is infinitesimal of higher order and can be ignored. The second term is only related to receiver phase noise and has the autocorrelation matrix

$$
\begin{aligned}
& E\left[j^{2} \mathbf{U}^{H} \bar{\Theta} \mathrm{HV} \tilde{x} \tilde{x}^{H} \mathbf{V}^{H} \mathbf{H}^{H} \bar{\Theta}^{T} \mathbf{U}\right] \\
& =-\mathrm{U}^{H} E\left[\bar{\Theta} \mathrm{HH}^{H} \bar{\Theta}^{T}\right] \mathrm{U} \cdot E\left[|x|^{2}\right] \\
& =-\mathrm{U}^{H} E\left[\bar{\Theta}\left[\mathbf{h}_{1,}^{T}, \mathbf{h}_{2,}^{T}, \ldots, \mathbf{h}_{N_{R}}^{T} \cdot\right]^{T}\left[\mathbf{h}_{1 .}^{H}, \mathbf{h}_{2,}^{H}, \ldots, \mathbf{h}_{N_{R}}^{H} .\right] \bar{\Theta}^{T}\right] \mathrm{U} \cdot E\left[|x|^{2}\right]
\end{aligned}
$$

where $E\left[|x|^{2}\right]=P / r$, and the channel matrix is assumed to be known, $\mathrm{H}=\left[\mathrm{h}_{1,}^{T}, \mathrm{~h}_{2,}^{T}, \ldots, \mathbf{h}_{N_{R}}^{T}\right]^{T}$. Since phase noises are mutually independent, i.e., for $i \neq j, E\left[\theta_{i} \theta_{j}^{*}\right]=0$, the further simplification is given by

$$
\begin{aligned}
& -\mathrm{U}^{H} E\left[\bar{\Theta}\left[\mathbf{h}_{1 .}^{T}, \mathbf{h}_{2 .}^{T}, \ldots, \mathbf{h}_{N_{R}}^{T} \cdot\right]^{T}\left[\mathbf{h}_{1 .}^{H}, \mathbf{h}_{2 \cdot,}^{H}, \ldots, \mathbf{h}_{N_{R}}^{H} . \bar{\Theta}^{T}\right] \mathrm{U}\right. \\
& =-\mathrm{U}^{H} \operatorname{diag}\left\{E\left[\left\|\mathrm{~h}_{1} .\right\|^{2}\right] \sigma_{\theta_{1}}^{2}, E\left[\left\|\mathrm{~h}_{2} .\right\|^{2}\right] \sigma_{\theta_{2}}^{2}, \ldots, E\left[\left\|\mathrm{~h}_{N_{R}} .\right\|^{2}\right] \sigma_{\theta_{N_{R}}}^{2}\right\} \mathrm{U}
\end{aligned}
$$


where $\sigma_{\theta_{l}}^{2}=E\left[\theta_{l}^{2}\right]$ is noise variance. Since $\mathrm{U}$ is unitary matrix, the noise power introduced by receiver phase noise is given by

$\operatorname{tr}\left\{\mathrm{U}^{H} \operatorname{diag}\left\{E\left[\left\|\mathrm{~h}_{1} .\right\|^{2}\right] \sigma_{\theta_{1}}^{2}, E\left[\left\|\mathrm{~h}_{2} .\right\|^{2}\right] \sigma_{\theta_{2}}^{2}, \ldots, E\left[\left\|\mathrm{~h}_{N_{R}} .\right\|^{2}\right] \sigma_{\theta_{N_{R}}}^{2}\right\} \mathrm{U}\right\} \cdot E\left[|x|^{2}\right]=\frac{P}{r} \sum_{l=1}^{N_{R}} E\left[\left\|\mathrm{~h}_{l}\right\|^{2}\right] \sigma_{\theta_{l}}^{2}$

If the channel is independent and identically distributed and Rayleigh faded, and $h_{i j} \sim$ $\mathcal{C N}(0,1)$, then $E\left[\left|h_{i j}\right|^{2}\right]=1$, and (52) is rewritten as

$$
\operatorname{tr}\left\{\mathrm{U}^{H} \operatorname{diag}\left\{E\left[\left\|\mathrm{~h}_{1} .\right\|^{2}\right] \sigma_{\theta_{1}}^{2}, E\left[\left\|\mathrm{~h}_{2} .\right\|^{2}\right] \sigma_{\theta_{2}}^{2}, \ldots, E\left[\left\|\mathrm{~h}_{N_{R}} .\right\|^{2}\right] \sigma_{\theta_{N_{R}}}^{2}\right\} \mathrm{U}\right\} \cdot E\left[|x|^{2}\right]=\frac{N_{T} P}{r} \sum_{l=1}^{N_{R}} \sigma_{\theta_{l}}^{2}
$$

The third term in (49) related only to the transmitter phase noise, whose autocorrelation matrix is calculated by

$$
\begin{aligned}
& E\left[j^{2} \mathrm{U}^{H} \mathrm{H} \bar{\Phi} \mathrm{V} \tilde{x} \tilde{x}^{H} \mathrm{~V}^{H} \bar{\Phi}^{T} \mathrm{H}^{H} \mathrm{U}\right] \\
= & -\mathrm{U}^{H} E\left[\mathrm{H} \bar{\Phi} \bar{\Phi}^{T} \mathrm{H}^{H}\right] \mathrm{U} \cdot E\left[|x|^{2}\right] \\
= & -\mathrm{U}^{H} E\left[\left[\mathrm{~h}_{\cdot 1} \phi_{1}^{2}, \mathrm{~h} \cdot{ }_{\cdot 2} \phi_{2}^{2}, \ldots, \mathrm{h}_{\cdot N_{T}} \phi_{N_{T}}^{2}\right]\left[\mathrm{h} \cdot 1, \mathrm{~h} \cdot 2, \ldots, \mathrm{h}_{N_{T}}\right]^{H}\right] \mathrm{U} \cdot E\left[|x|^{2}\right]
\end{aligned}
$$

For Rayleigh fading channels

$$
\operatorname{tr}\left\{\mathrm{U}^{H} E\left[\left[\mathrm{~h} \cdot 1 \phi_{1}^{2}, \mathrm{~h} \cdot 2 \phi_{2}^{2}, \ldots, \mathrm{h} \cdot \mathrm{N}_{\mathrm{T}} \phi_{N_{T}}^{2}\right]\left[\mathrm{h} \cdot 1, \mathrm{~h} \cdot 2, \ldots, \mathrm{h} \cdot \mathrm{N}_{\mathrm{T}}\right]^{H}\right] \mathrm{U}\right\} \cdot E\left[|x|^{2}\right]=N_{R} \sum_{l=1}^{N_{T}} \sigma_{\phi_{l}}^{2} \cdot E\left[|x|^{2}\right]
$$

Then the average SINR on the receiving side

$$
\begin{aligned}
S I N R & =\frac{E\left[P_{R}\right]}{E\left[P_{I}\right]+N_{0}} \\
& \approx \frac{\sum_{i=1}^{r} E\left[\lambda_{i}^{2}\right]-N_{T} \sum_{l=1}^{N_{R}} \sigma_{\theta_{l}}^{2}-N_{R} \sum_{l=1}^{N_{T}} \sigma_{\phi_{l}}^{2}}{N_{T} \sum_{l=1}^{N_{R}} \sigma_{\theta_{l}}^{2}+N_{R} \sum_{l=1}^{N_{T}} \sigma_{\phi_{l}}^{2}+N_{0} / E\left[|x|^{2}\right]} \\
& =\frac{1-\frac{1}{N_{R}} \sum_{l=1}^{N_{R}} \sigma_{\theta_{l}}^{2}-\frac{1}{N_{T}} \sum_{l=1}^{N_{T}} \sigma_{\phi_{l}}^{2}}{\frac{1}{N_{R}} \sum_{l=1}^{N_{R}} \sigma_{\theta_{l}}^{2}+\frac{1}{N_{T}} \sum_{l=1}^{N_{T}} \sigma_{\phi_{l}}^{2}+N_{0} /\left(E\left[|x|^{2}\right] N_{R} N_{T}\right)} \\
& \approx \frac{1-E\left[\sigma_{\theta_{l}}^{2}\right]-E\left[\sigma_{\phi_{l}}^{2}\right]}{E\left[\sigma_{\theta_{l}}^{2}\right]+E\left[\sigma_{\phi_{l}}^{2}\right]+r /\left(\gamma_{0} N_{R} N_{T}\right)}
\end{aligned}
$$

where $\gamma_{0}=P / N_{0}$. It follows that the average SINR is subject to the average of the variance of phase noises at both transmitters and receivers.

\subsection{Discuss on synchronization}

The suppression of phase noise should be done by two ways: on one hand, making the noise as low as possible during system design; on the other hand, suppressing and eliminating the effects of phase noise by means of algorithms.

The MIMO system we studied here is a narrowband system. When receive beamforming is used, the RF signal from the transmitter can be used as a reference signal in order to realize 
the synchronization in frequency and phase; whereas when only transmit beamforming is used, frequency synchronization can be realized by synchronizing all transmitter carrier frequencies with receiver, or using an extra transmitter as reference frequency. In the latter case, a time-division periodical synchronization scheme is required in order to correct the phase error induced by propagation. However, phase noise during the signal transmission can not be eliminated, especially when joint transmit/receive beamforming (linear precoding) is applied. Therefore, minimizing the noise during circuit design is a better way to solve this problem.

In a distributed MIMO system with independent transmitter and receiver clocks, a common independent RF reference signal is necessary. The meaningful phase noise estimation and suppression can only be made for broadband MIMO systems.

\section{Simulation results}

Referring to (6), the variance of Wiener phase noise will increase linearly with time. Assuming phase noises are independent and identically distributed, carrier frequency $f_{c}=2 \mathrm{GHz}$, and the constant of an open-loop VCO $c_{v c o}=10^{-19}$, the variation of phase noise variance $\sigma_{\theta}^{2}$ with time is shown in Fig. 6 for a free-running oscillator. At $t=0.5 \mathrm{~ms}, \sigma_{\theta}^{2} \approx 0.01$, and if the average of $E\left[\sigma_{\theta_{l}}^{2}\right] \approx 0.01$, the corresponding SINR will be less than $20 \mathrm{~dB}$. It should be emphasized that different from application of single-layer beamforming (for point to point communication), application of precoding demands on electronic devices with a better noise characteristics. It is because independent phase noises result in not only the reduction of signal's active power, but also the interference among parallel channels.

Fig. 7 describes the phase noise characteristics of a PLL synthesizer. Within a time duration less than $100 \mathrm{~ms}$, phase noise at the PLL output is determined by $\beta(t)$, which is one component of the variance bounded multi-dimension Ornstein-Uhlenbeck process. Therefore, the variance of $\alpha_{p} l l(t)$ approximates a constant. In a relative large observation time range, $\alpha_{p} l l(t)$ will depend on the phase noise of the reference signal $\alpha_{p} l l(t)$. Furthermore, with a VCO of the same quality, PLL output signal has a much better noise characteristic, and better performance can be obtained by the system.

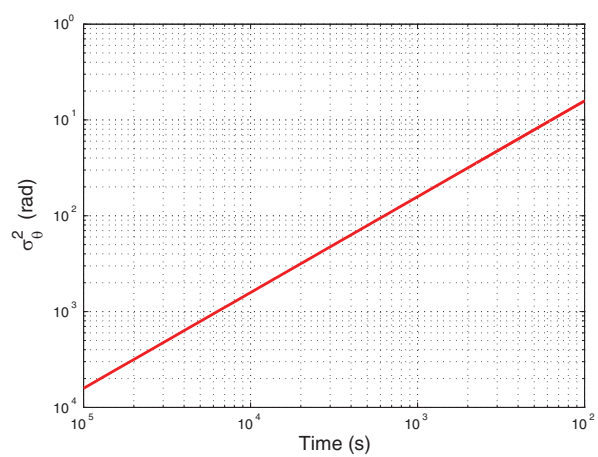

(a) Wiener noise: noise power increase linearly with time

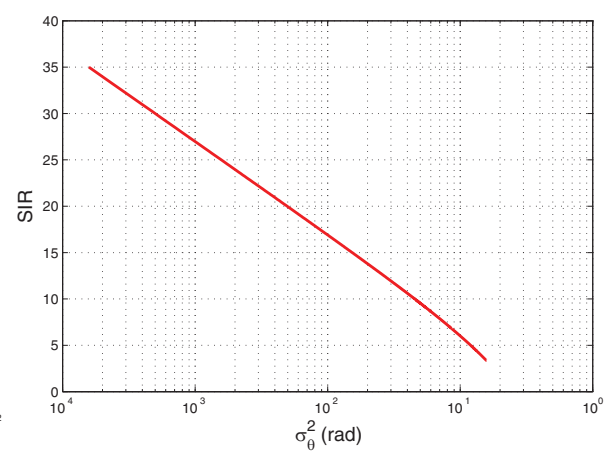

(b) Wiener noise: SINR decreases with the increased noise power

Fig. 6. Simulation for a free-running oscillator 


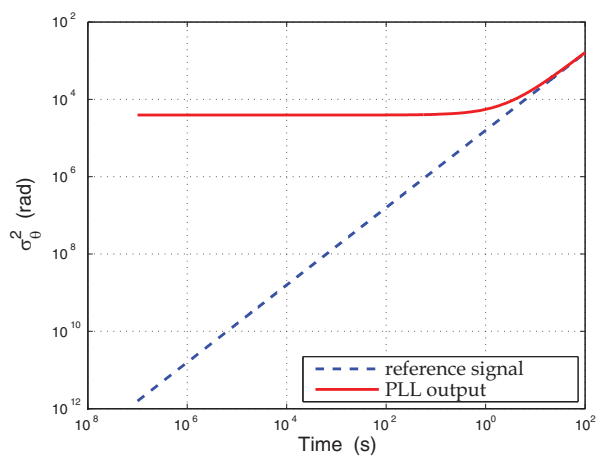

(a) PLL phase noise: noise power varies with (b) PLL phase noise: SINR decreases with the time, for $c_{\text {ref }}=10^{-25}, c_{v c o}=10^{-19}$

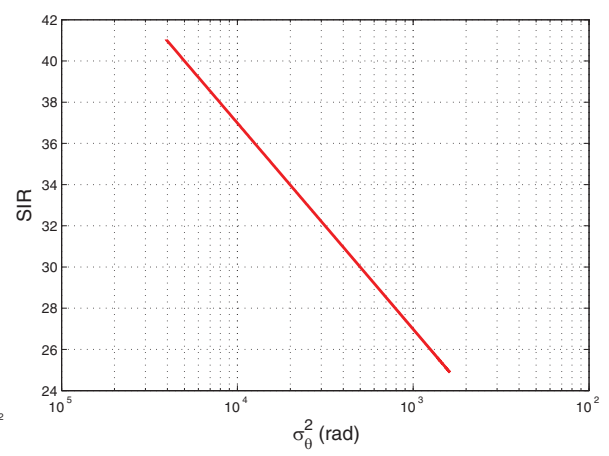

increased noise power

Fig. 7. Simulation for a PLL synthesizer

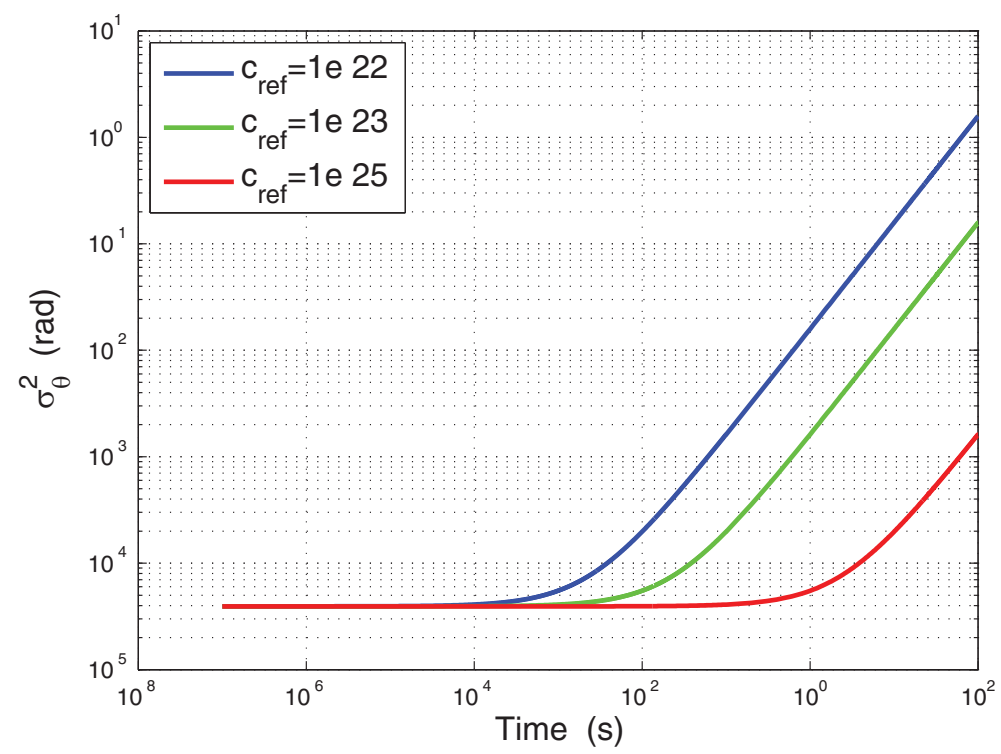

Fig. 8. Comparison of phase noise variance of PLL with difference reference crystal

Fig. 8 compares the phase noise variance of PLL synthesizers using reference clocks of different quality. Obviously, the better the quality of the clock, the better the stability of the PLL is.

In the literature (Mudumbai et al., 2007) the influence of independent phase noises on a distributed wireless sensor network using transmit beamforming has been studied. Application of transmit/receive beamforming can bring the potential power gain, but requires the carrier synchronization in phase and frequency. In addition, if single-layer beamforming is used(transmit/receive beamforming), independent phase noises at transmitter/receiver will reduce the power gain, but no interference is introduced; whereas with precoding 
(joint transmit/receive beamforming), extra interference exits among sub-streams and further worsens the receive SNR.

\section{Conclusions and future research}

In this chapter, we have investigated the effects of phase noise in centralized and distributed narrowband MIMO systems, and discussed the feasibility of phase and frequency synchronization. In centralized narrowband MIMO systems, the effects of phase noise is similar to that in simple point-to-point communications, except that phase noise may affect the accuracy of channel estimation and thus the channel capacity is overestimated (Taparugssanagorn \& Ylitalo, 2009).

The simulation results have shown that in distributed MIMO systems, independent phase noises not only give rise to the reduction of power gain provided by transmit/receive beamforming, but also induce the interference among parallel channels provided by precoding. The signal-to-interference ratio decreases rapidly when the average interference power increases. Therefore, in decentralized MIMO systems more precise devices are demanded, which contradicts the low cost we expected. Furthermore, phase synchronization in a distributed narrowband system raises the overhead cost. Even so, during data transmission phase noise can not be corrected.

For a broadband system, overhead for phase noise estimation and mitigation is also a challenge. It is a hot topic at the moment as well as in the future.

\section{References}

Bittner, S., Rave, W. \& Fettweis, G. (2007). Phase noise suppression in ofdm with spatial multiplexing, VTC-2007 Spring, pp. 1826-1830.

Demir, A. (1998). Phase noise in oscillators:dae's and colored noise sources, Proc. IEEE/ACM Int. Conf. CAD, pp. 170-177.

Demir, A., Mehrotra, A. \& Roychowdhury, J. (2000). Phase noise in oscillators: A unifying theory and numerical methods for characterisation, IEEE Trans. on Circuits Syst. 47(5): 655-674.

Kim, Y. W. \& Yu, J. D. (2008). Phase noise model of single loop frequency synthesizer, IEEE Trans. on Broadcasting 54(1): 112-119.

Leeson, D. B. (1966). A simple model of feedback oscillator noise spectrum, Proc. of the IEEE 54(2): 329-330.

Liu, P., Wu, S. \& Bar-Ness, Y. (2006). A phase noise mitigation scheme for mimo wlanss with spatially correlated and imperfectly estimated channels, IEEE Commun. Letters 10(3): 141-143.

Mehrotra, A. (1999). Simulation and modeling techniques for noise in radio frequency integrated circuits, PhD thesis, Univ. California, Berkeley.

Mehrotra, A. (2002). Noise analysis of phase-locked loops, IEEE Trans. on Circuits Syst. 49(9): 1309-1316.

Mudumbai, R., Barriac, G. \& Madhow, U. (2007). On the feasibility of distributed beamforming in wireless networks, IEEE Trans. on Wireless Commun. 6(4): 1754-1763.

Petrovic, A., Wolfgang, R. \& Fettweis, G. (2007). Effects of phase noise on ofdm systems with and without pll: characterization and compensation, IEEE Trans. on Commun. 55(8): 1607-1616. 
Robins, W. P. (1982). Phase Noise in Signal Sources (Theory and Applications), Peter Peregrinus Ltd., London.

Rohde, U. L. \& Netwkirk (2000). RF/Microwave Circuit Design for Wireless Applications, John Wiley \& Sons. Inc.

Schenk, T. C. W., Tao, X.-J., Smulders, P. F. M. \& Fledderus, E. R. (2004). Influence and suppression of phase noise in multi-antenna ofdm, VTC-2004 Fall, Vol. 2, pp. 1443-1447.

Taparugssanagorn, A. \& Ylitalo, J. (2009). Characteristics of short-term phase noise of mimo channel sounding and its effect on capacity estimation, IEEE Trans. on Instru. $\mathcal{E}$ measuI. 58(1): 196-201.

Tse, D. \& Viswanath, P. (2004). Fundamentals of Wireless Communication, Cambridge University Press.

Zhang, W. (2010). Phase Noise in MIMO systems, PhD thesis, Tsinghua University, Beijing. 


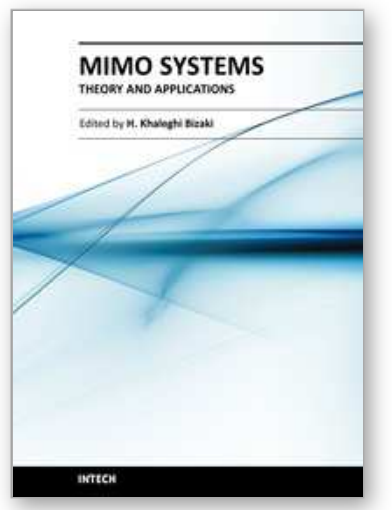

\author{
MIMO Systems, Theory and Applications \\ Edited by Dr. Hossein Khaleghi Bizaki
}

ISBN 978-953-307-245-6

Hard cover, 488 pages

Publisher InTech

Published online 04, April, 2011

Published in print edition April, 2011

In recent years, it was realized that the MIMO communication systems seems to be inevitable in accelerated evolution of high data rates applications due to their potential to dramatically increase the spectral efficiency and simultaneously sending individual information to the corresponding users in wireless systems. This book, intends to provide highlights of the current research topics in the field of MIMO system, to offer a snapshot of the recent advances and major issues faced today by the researchers in the MIMO related areas. The book is written by specialists working in universities and research centers all over the world to cover the fundamental principles and main advanced topics on high data rates wireless communications systems over MIMO channels. Moreover, the book has the advantage of providing a collection of applications that are completely independent and self-contained; thus, the interested reader can choose any chapter and skip to another without losing continuity.

\title{
How to reference
}

In order to correctly reference this scholarly work, feel free to copy and paste the following:

Wei Zhang, Xiujun Zhang, Shidong Zhou and Jing Wang (2011). Analysis and Mitigation of Phase Noise in Centralized/De-centralized MIMO Systems, MIMO Systems, Theory and Applications, Dr. Hossein Khaleghi Bizaki (Ed.), ISBN: 978-953-307-245-6, InTech, Available from: http://www.intechopen.com/books/mimosystems-theory-and-applications/analysis-and-mitigation-of-phase-noise-in-centralized-de-centralized-mimosystems

\section{INTECH}

open science | open minds

\section{InTech Europe}

University Campus STeP Ri

Slavka Krautzeka 83/A

51000 Rijeka, Croatia

Phone: +385 (51) 770447

Fax: +385 (51) 686166

www.intechopen.com

\section{InTech China}

Unit 405, Office Block, Hotel Equatorial Shanghai

No.65, Yan An Road (West), Shanghai, 200040, China

中国上海市延安西路 65 号上海国际贵都大饭店办公楼 405 单元

Phone: +86-21-62489820

Fax: $+86-21-62489821$ 
(C) 2011 The Author(s). Licensee IntechOpen. This chapter is distributed under the terms of the Creative Commons Attribution-NonCommercialShareAlike-3.0 License, which permits use, distribution and reproduction for non-commercial purposes, provided the original is properly cited and derivative works building on this content are distributed under the same license. 